\title{
Prólogo
}

\section{Capital Social y Capital}

\author{
Gardy Augusto Bolívar Espinoza \\ Universidad Autónoma de México - Sede Azcapotzalco, Ciudad de México, México. \\ Email: abe88@hotmail.com
}

\section{Antonio Elizalde Hevia}

Polis, Santiago de Chile. Email: aelizalde@ubolivariana.cl

La perspectiva teórica del Capital, del siglo XIX, aparece alejada del capital social del siglo XXI. Ambas, con la misma raíz, sin embargo, pareciera que no se tocan, ni teórica ni prácticamente. Quizás, sería pertinente escudriñar esta relación.

Desde la década de los noventa, el tema del "capital social” ha sido visto por muchos estudiosos de la realidad social contemporánea como una propuesta promisoria, transversal y hegemónica. Tanto desde la economía, las ciencias morales, la ciencia y la sociología política, expertos de diversas instituciones internacionales han concurrido a este campo en busca de soluciones para el diseño de políticas públicas, que es donde parece brillar más este concepto.

El “capital social”, sin embargo, -al contrario de su homónimo, el capital de Marx- no tiene una sola vertiente epistemológica ni posee una sola biblia que lo anide y, más bien, se expresa en múltiples vulgatas que se esfuerzan por encontrar su significado. Dentro de estas diversas interpretaciones, sin embargo, el neoliberalismo económico, la perspectiva del rational choice y el individualismo metodológico parecieran tener un papel especial. Aunque no es menos cierto que el tema del capital social también ha sido abordado por liberales y por movimientos sociales progresistas, que ven en él modos de acceder a una mayor equidad y a más justas y solidarias relaciones sociales.

Teñido de aportes teóricos y metodológicos novedosos, el capital social no está exento de referencias a clásicos antiguos y cercanos. Este hecho le da mayor amplitud y riqueza, pero también contribuye a su oscuridad: definiciones vagas o, simplemente, falta de definición, situación que afecta seriamente los intentos de cuantificación. El “Capital social” se usa pero no se está muy claro en qué se entiende por él. Probablemente porque, en su comprensión, concurre oblicuamente la sociología clásica en temas tradicionales de la economía; y, ésta vuelve a reconsiderar su relación con la ciencia política. De ahí que no es extraño que se cite a menudo a Aristóteles, Rousseau, Smith, Tocqueville, y Durkheim, pero, poco se cita a Marx. 
Dos son las corrientes en que se puede ordenar el amplio campo del capital humano: las "definiciones estructurales" fundamentalmente referidas a Bourdieu y Coleman, y las definiciones culturales cuyo mejor representante sería Putnam. La primera se ubica, según Bourdieu, en "el agregado de recursos reales o potenciales que se vinculan con la posesión de una red duradera de relaciones más o menos institucionalizadas de conocimiento y reconocimiento mutuo": acceso a la información, obligaciones que se desprenden de la confianza mutua, o aprovechamiento de las normas sociales cooperativas. La referencia material de este enfoque se encuentra en las redes. Éstas tienen historia y continuidad, pueden estar cerradas, ser unidireccionales, simétricas, horizontales o, sus contrarios; los vínculos pueden ser fuertes o débiles o frecuentes, con puentes hacia otras redes, o volcados hacia su interior, etc.

La segunda, refiere a un fenómeno subjetivo: valores y actitudes cuyo centro es la confianza generalizada, vinculada a un "juicio moral" conectado con la "cultura política”. Se podría decir que para Putnam la virtud cívica es capital social. Los temas que recurren a este concepto son variados y amplios: la creación de Capital Humano; la efectividad de las instituciones democráticas; el desarrollo económico; y la acción colectiva. En las investigaciones sobre capital social éste aparece ya sea como variable dependiente o independiente: ya sea el conjunto de causas y procesos implicados en su formación, o sus consecuencias.

Uno de los intentos de aclarar el "capital social” sostiene que su denominación es retórica; el capital social sería sólo una "metáfora” o una metonimia. Sin embargo, no queda claro qué papel jugaría en el caso del capital social cada uno de los términos que componen este recurso lingüístico. Más bien, como indica la evidencia, lo social de este "nuevo" capital es un adjetivo, por lo demás no muy novedoso; afirmaría que el capital sería social, en oposición a otros capitales, los cuales: ¿no serían sociales? Se trataría de un "vehículo", pero no se define cuál es el "fundamento" que permitiría enriquecer el significado del "tenor”. Desde la CEPAL se intenta justificar así al Capital social como metáfora:

"Los recursos son instrumentalidades que pueden ser aplicadas a la consecución de ciertos fines. En cuanto tales, son acumulables, transferibles, y aplicables a fines productivos. Es la noción de "recurso" lo que fundamenta la metáfora de la asociatividad tratada como "capital”." (Atria (Consultor CEPAL), 2002). Es decir, lo que se entendería por capital social serían... recursos.

El "capital social” sería diferente al financiero y al de recursos materiales, donde la diferencia pareciera estribar en que este capital social tendría una existencia etérea mientras que los otros una "concreta" y, en aras de un realismo empírico pareciera ser adecuado darle la concreción del capital a las asociaciones humanas. Por su parte, El Capital, producto de la Crítica a la Economía Política, ha sido fuente simbólica y heurística que se impuso como referente sagrado o demoníaco y que se sostuvo en el 
centro de la disputa ideológica, práctica y científica por lo menos hasta el último cuarto del siglo XX. Sus repercusiones políticas son evidentes y, en economía, la relación valor-trabajo pareciera que tiene mucho que decir todavía. Sin embargo, en el plano de la sociología y de las políticas públicas, no aparecen claras sus posibles influencias. Habría que ver si esa ausencia es solamente aparente o si el tiempo ha relegado sus postulados a la crítica de los ratones.

El capitalismo de Marx es una forma histórica, y su concepción podría inscribirse como la de uno de los más relevantes fundadores de las ciencias sociales; pero se vería mal como fundador de la sociología. Por su parte, el "capital social” sería una forma de comprender aquello de lo social que no es ni estructura jurídica, ni política, ni económica, y de ver las relaciones sociales en sí mismas, como si fueran cosas, es decir, como sociología francesa clásica. Sin embargo, en Marx, el capital mercantil, el financiero, y el industrial son formas etéreas (abstractas), manifestaciones, metamorfosis de un mismo proceso del trabajo: el modo de producción capitalista. El capitalismo sería una forma, como las relaciones económico-sociales; de "pertenencia obligatoria", se desenvuelven en una realidad presente determinada, única, diferente a otros modos de producción históricos.

Como lo plantea el Banco Mundial, el "capital social” se liga temáticamente a las "normas de confianza", "los valores", "las actitudes y redes" existentes entre personas e instituciones, en el marco de la "asociatividad" o de las "acciones colectivas y de cooperación”. En lo individual, se refiere al grado de integración con su entorno a partir de las relaciones más cercanas de una persona, familia o empresa. A nivel sectorial, la acción se visualiza a través de personas u organizaciones en un entorno ampliado con los poderes públicos: pertenencia a redes comunitarias, gremios, asociaciones empresariales, etc. Finalmente, en la dimensión colectiva o nacional, el capital social se entiende como un acervo de una sociedad global. En ese sentido, el concepto de Capital Social agrupa nociones de confianza, asociatividad, conciencia cívica y valores éticos predominantes que, en conjunto, facilitan o dificultan el desarrollo de una determinada sociedad.

En América Latina destacan varios programas de combate a la pobreza en los que destaca la presencia de CEPAL, del Banco Mundial y de universidades del continente y de Europa. La Conferencia internacional "Hacia un nuevo paradigma: Capital social y reducción de la pobreza en América Latina y el Caribe”, organizada por la Comisión Económica para América Latina (CEPAL) y la Universidad del Estado de Michigan en Santiago de Chile, en septiembre de 2001 representa una referencia obligada para los estudios de la región y en especial los del capital social.

Siete temas propuso el encuentro donde se abordó el análisis del capital social: su relación con el desarrollo, las políticas públicas, la pobreza urbana, la dimensión de género, el mundo rural y la sostenibilidad am- 
biental mediante perspectivas teóricas y analíticas, así como enfoques disciplinarios heterogéneos. Entre los principales desafíos futuros planteados, se encuentra la necesidad de explorar la formación y mantención de capital social en las sociedades de América Latina y el Caribe, que son altamente desiguales y segmentadas. Asimismo, de examinar las mejores formas de eslabonamiento entre organizaciones de base y Estado para eliminar aspectos negativos del desarrollo latinoamericano, como son el clientelismo y la corrupción. En ese desafío adquiere extrema importancia el fortalecimiento de los actores sociales más débiles y el rendimiento de cuentas de la gestión pública en sus niveles municipales, regionales y nacionales. Entre los actores principales que hay que considerar, se encuentran las mujeres pobres urbanas y rurales, así como grupos rurales e indígenas largamente excluidos de los procesos de desarrollo. (CEPAL, 2010).

La convocatoria realizada por Polis en este número logró recibir un interesante conjunto de aportes teóricos, metodológicos, críticos o informativos, respecto del campo del capital social, las cuales se incluyen en nuestra sección “Lente de Aproximación”, que se inicia con el artículo de Gardy Augusto Bolívar Espinoza y Rogelio Caloca Osorio, titulado “Capital social y humano en convergencia. Asentamientos irregulares de Milpa Alta"; allí se afirma que el capital social, si bien corresponde a una categoría nacida de la economía política clásica y, en particular, empleada por Karl Marx, se puede presentar en su versión contemporánea a través de los modelos de combinación del capital físico y el capital humano en el contexto de la teoría de la economía del bienestar; en cuyo trasfondo subyace el capital social. Los autores exponen la cuestión conceptual y al capital humano en un modelo de crecimiento de Convergencia. Se consideran los niveles de escolaridad promedio en los asentamientos irregulares de la Delegación Milpa Alta en el Distrito Federal entre 2000-2005, y se proyectan las tasas al 2020 que mantiene los resultados de Convergencia; obtenidos en el modelo para el 2000-2005, demostrando la relación entre valortrabajo educción y crecimiento. Considerando las unidades de trabajo simple agregadas en trabajo complejo, es posible dar razón de las transformaciones de un análisis desde Marx a las consideraciones estipuladas por Schultz en la formación de capital humano. El análisis del capital humano presentado no altera las condiciones que se reflejan en el modelo original de Solow, puesto que al agregar el capital humano, la conclusión que se tiene es que las naciones, regiones o localidades son más ricas unas que las otras debido a que dedican una mayor parte de su tiempo al aprendizaje de habilidades para el trabajo, entre otras cosas. Además, provee los mecanismos para saber si en el caso estudiado los asentamientos irregulares están mejorando en sus condiciones educativas. El estudio muestra que las mujeres y los hombres de este lugar sí están mejorando sus condiciones, pues pasan la prueba tanto de $\sigma$-Convergencia como de $\beta$-Convergencia, es decir, sus tasas de escolaridad promedio convergen y con ello, los más rezagados experimentarán un mayor crecimiento de sus tasas de escolaridad que los que están en mejor situación. Pese a que existen estímulos económicos suficientes en la zona para que un buen porcentaje de hombres dejen de estudiar, esto bien puede verse compensado por las crecientes tasas de 
incorporación de las mujeres a ciclos educativos más elevados. Si bien el proceso de generación de capital humano es atractivo para el desarrollo de una nación, región o localidad, también es necesario atender que sin los empleos necesario que contraten a dicha fuerza de trabajo capacitada el proceso no se cerrará y se tendrán personas con alto grado de preparación y con sueldos y salarios paupérrimos, o que se encuentren desempleados o desempeñándose en alguna actividad diferente a sus estudios. Si este es el caso, el modelo de capital humano falla en su cierre con la economía del trabajo.

En un segundo trabajo de Gardy Augusto Bolívar Espinoza, esta vez en coautoría con Leonel Flores Vega, titulado "Discutir el campo del capital social desde un enfoque transdisciplinario", se exponen algunos antecedentes sobre el origen y las vertientes actuales del campo del capital social en las ciencias sociales en tanto instrumento analítico de la realidad social. Este artículo trata de los fundadores y de sus posiciones así como los trabajos emprendidos desde las organizaciones internacionales, especialmente, el Banco Mundial. Brevemente, se refiere a dimensiones e indicadores, señalando los principales aportes y desafíos. El objetivo del trabajo es mostrar cómo el capital social se formó como un campo de estudio, iniciado con algunos antecedentes en las ciencias sociales, seguido de los fundadores formales y organismos internacionales. Se sostiene que -a pesar de la falta de acuerdo y claridad del concepto -la que permitiría decir cualquier cosa de cualquier tema-, el campo del capital debe considerarse como un espacio contradictorio y prometedor de las nuevas ciencias sociales. Se ha planteado más bien que es un concepto multidimensional, dado que incorpora diferentes niveles y unidades de análisis, y los campos que se ven influenciados son diversos. Además, varían de acuerdo a cada formulación teórica y experiencia que se tiene, debido a la evolución de las relaciones sociales y su vínculo con las organizaciones informales e instituciones formales. En el artículo se comparte la idea que el capital social incluye "el ambiente social y político que conforma la estructura social”: relaciones y estructuras institucionales formalizadas que permiten el desarrollo de normas a través del régimen político y el gobierno, el sistema judicial y la aplicación del el derecho, y las libertades civiles y políticas. El enfoque transdisciplinario no sólo toma en cuenta las virtudes y los vicios del capital social, y la importancia de la formación de relaciones dentro y entre comunidades, sino también reconoce que la capacidad de varios grupos sociales para actuar en su propio interés depende crucialmente del apoyo (o falta de apoyo) que reciban del Estado y del sector privado. De la misma manera, el Estado depende de la estabilidad social y del apoyo popular. En resumen, el desarrollo económico y social prospera cuando los representantes del Estado, del sector empresarial y de la sociedad crean foros a través de los cuales se puedan identificar y alcanzar metas comunes. En las investigaciones realizadas sobre capital social, no se ha logrado establecer indicadores comunes. No se han dirigido esfuerzos en los estudios para medir el capital social de una forma común; por el contrario, se han anclado a indicadores utilizados en otros índices y mediciones que van desde medidas de confianza, tendencias políticas o movilidad social. Los resulta- 
dos empíricos dejan pendiente una pregunta crucial: ¿cómo producir capital social? Tan pronto nos interrogamos sobre cómo potenciar el capital social y fortalecer a la sociedad, desembocamos en un argumento circular: más capital social produce más sociedad y, de manera recíproca, una sociedad fuerte crea más capital social. Por lo tanto, no es el capital social el remedio milagroso que resucitará a la sociedad activa, cabe más bien suponer lo contrario: donde es débil la sociedad, también es débil la construcción de capital social.

En un artículo que lleva por título "La variable ideológica como elemento central para la constitución y desarrollo de capital social" Jorge Brower Beltramin analiza uno de los temas más relevantes en el campo del capital social desde el ámbito de la cultura y de una perspectiva cognitiva (capital social cognitivo). Su objeto es la ideología en la constitución y desarrollo de capital social, entendida como "valores, creencias y normas”, apoyándose en los referentes teóricos de Putnam, Bourdieu, Mauss, Granoveter y Coleman. Lo que le preocupa, desde una perspectiva también ideológica, es: la instalación descontrolada del aparato neoliberal en la sociedad planetaria que ha creado una relación tensa entre un neo mercantilismo y ciertas reservas de capital social. Por una parte, la mercadización, promovida por las tendencias políticas de la derecha actual, se orienta por un consumismo hiperactivo irracional; por la otra parte, los sistemas de significación desde los cuales se construye el capital social protegerían un nomos esencial que resguarda el sentido antropológico de la existencia del hombre en espacios naturales y culturales en que ha vivido y construido con el tiempo. Concluye que la existencia de sistemas ideológicos con capacidad de adaptación y, al mismo tiempo, vigorosos y densos respecto a sus contenidos fundamentales, representan verdaderas fuerzas de resistencia en relación a un nuevo orden social articulado desde las bases del neoliberalismo económico.

Alejandro Garcés en su artículo "Comercio inmigrante y economías étnicas: síntesis y críticas de los debates vigentes” presenta una investigación sobre los flujos migratorios contemporáneos, un campo poco investigado e insuficientemente puesto en relación con las vidas cotidianas de las migrantes. El autor aborda, en primer término, los enfoques teóricos y las dimensiones de la "economía étnica" expuestas por Wilson, Portes y Martin, en el enclave cubano en Miami y, posteriormente se refiere en forma amplia a autores europeos y estadounidenses, en especial a los análisis de los negocios creados por inmigrantes en las sociedades de destino SurNorte. La economía étnica referida a la actividad económica por parte del individuo migrante, utilizando recursos étnicos en la instalación y gestión de los negocios, y en definitiva por una restrictiva circulación en torno a una determinada identidad o minoría cultural. Esta aproximación da cuenta de la forma en la que el sector migrante y los grupos étnicos buscan alternativas de inserción en la sociedad moderna, que observan la inauguración de lo étnico como objeto de un comercio, o si se quiere, que transforman lo étnico en objeto de consumo. Se destaca la articulación tanto de recursos de clase como étnicos, los que repercuten en el incremento o reducción del 
flujo migratorio a una determinada localidad. El autor abre la noción de economía étnica -reducción a un colectivo o minoría- convirtiéndola en un espacio económico no sólo de migrantes sino también de nativos; se inaugura el objeto de lo étnico como espacio de comercio o en objeto de consumo. Pueden señalarse dos elementos que caracterizan este tipo de economía: el empresario étnico o migrante; y la dependencia o utilización de recursos étnicos como especie de acervo cultural. Garcés critica a esta "burbuja” culturalista como fenómeno etno-cultural dentro de un vacío económico e institucional que hace posible la distinción, bastante útil, entre recursos étnicos y recursos de clase. Los recursos étnicos serían el conjunto de elementos o factores socioculturales que estarían a la base del surgimiento y sostenimiento de los negocios: redes sociales étnicas para la transmisión rápida de información, formación, consejos o experiencia, amistad, matrimonio, confianza, capital social, factores culturales, religión, lengua, valores y actitudes empresariales, asociaciones de crédito rotativas, solidaridad, y la ideología de solidaridad étnica. También podrían considerarse las prácticas etno-culturales, ideologías étnicas, redes sociales e instituciones étnicas. Paralelamente los recursos de clase, como las distintas formas de capital en posesión de un grupo: ya sea éste financiero, humano, cultural o material. Estos planteamientos se amplían y adecúan a los nuevos procesos producidos en América del Sur. En este caso se trata especialmente el caso chileno, donde la normativa que regula la vida de los migrantes corresponde a los setenta. Pero donde la flexibilización de los mercados de trabajo debido al marco de reformas económicas liberales, en cierta medida, discrimina a los inmigrantes menos calificados y peor remunerados y los fuerza a convertirse en "micro-empresarios". Su postura recurre a perspectivas y enfoques teóricos como: el culturalista, ecológico, estructuralista, desde las oportunidades, interaccionista y el modelo del encajado mixto.

"Capital social y clientelismo: otra limitación para el control social" se titula el trabajo de Mauricio García Ojeda, el cual afirma que desde mediados de la década de los noventa los estudios sobre capital social se orientan a sus consecuencias positivas y, en menor medida, a las negativas. Entre los aspectos críticos destaca el cuestionamiento, -además de su incapacidad explicativa- al carácter del "capital”, al contenido que pudiera adjudicársele. Pareciera que el capital social más que un concepto o una categoría sería una analogía de la cual se derivarían hipótesis cuestionables. El autor encara una de las manifestaciones del "lado oscuro" del capital social: la coacción política en relaciones clientelares. Se trata de observar el uso del capital social respecto en la neutralización de las acciones de control de los ciudadanos mediante la amenaza de suspensión de la asistencia desarrollada a partir de la implementación de capital social, si los mecanismos de control ciudadano sobrepasan los límites que permiten los operadores políticos. El capital social pudiera limitar el ejercicio del control social. El artículo expone algunas distinciones analíticas sobre el capital social desde la relación ciudadanos/políticos por intermedio de la teoría principal-agente y para explicar la asimetría de poder entre ambos se hace uso de la teoría del intercambio disputado formulada por la economía política radical. La tesis central es que el capital favorece la dominación de 
los obreros en los mercados de trabajo capitalistas y el capital social puede favorecer la dominación de los ciudadanos en las relaciones clientelares. El capital social se entiende como recursos tales como información, obligaciones de reciprocidad y normas expresadas en sanciones efectivas. En la relación entre capital social y clientelismo se propone que en las relaciones clientelares uno de los recursos que se intercambia es la información como capital social. Además, para que se produzcan las expectativas necesarias para que el intercambio de este recurso se mantenga en el tiempo, es necesaria la concurrencia de obligaciones de reciprocidad que también son un recurso de capital social disponible gracias a que los implicados están insertos en la relación clientelar como una red de relaciones de mutuo beneficio. Finalmente, se afirma que desde las ciencias sociales es necesario proponer e impulsar mejores diseños institucionales para hacer más efectivo el control social que permita una mejor gobernanza democrática.

Ignacio Madero Cabib y Claudia Mora del Valle presentan el trabajo titulado "Capital Social e Inclusión Laboral. Una aproximación a las trayectorias de ascendencia laboral de migrantes peruanos en Chile”. Señalan que desde la década de los noventa la migración peruana en Chile crece en forma exponencial, pero sólo recientemente tiene la atención de los investigadores sociales. Los autores presentan algunos de los resultados de un trabajo de investigación empírica basado en entrevistas a 32 migrantes peruanos de ambos sexos y que han experimentado un reciente y relativo éxito en su inclusión laboral. Entre las conclusiones de este estudio se muestra que la permanencia de matrices de estratificación social chilenas afectan los procesos de inclusión de los migrantes peruanos y condiciona sus oportunidades de vida y sus trayectorias laborales orientándolos a utilizar vías informales de inclusión en el mercado del trabajo. Los autores enfatizan la existencia de dinámicas de inclusión social determinadas por el sistema de estratificación y por criterios extra-laborales situación que deriva en su dependencia de la formalización de capital social como mecanismo de inclusión. La segmentación laboral de los inmigrantes peruanos en nichos precarios no considera su capital cultural a causa de la rigidez del mercado laboral que no está en condiciones de generar mecanismos abiertos de inclusión. En estas condiciones los migrantes deben recurrir a tácticas y estrategias para su inclusión formal e informal frente a los obstáculos que supone el origen nacional y la percepción racial. En Chile se ha edificado una forma particular de estratificación que afecta generalmente a los inmigrantes peruanos. Aquí, el origen nacional, la clase, la etnia, el género o la percepción racial operan como obstaculizadores de la inclusión laboral, dificultando el acceso a ocupaciones coherentes con el capital cultural de cada persona. Sin embargo, como plantea Archer, los inmigrantes son portadores de una capacidad agencial que logra traspasar las constricciones institucionales, generando posibilidades de movilidad ocupacional a través del despliegue de tácticas y estrategias para eludir las barreras presentadas a los mecanismos de inclusión. La evaluación reflexiva de los inmigrantes es el antecedente necesario. La ascendencia laboral de los inmigrantes puede tener lugar cuando acciones y reacciones se despliegan ante el reconoci- 
miento de oportunidad de inclusión al mercado del trabajo. El siguiente paso, es la exploración de las circunstancias objetivas y subjetivas que facilitan la apertura de esta condición de posibilidad.

El artículo de Mariano Scheinsohn y Cecilia Cabrera, titulado "Más allá del capital social. La producción de hábitat popular en Buenos Aires", presenta la disparidad de definiciones y el uso ambiguo del concepto de capital social que afecta su capacidad interpretativa y explicativa. Los autores lo entienden como la valorización de un recurso ligado a la construcción de una voice pública, a la conformación de un liderazgo interno, y a una legitimidad externa frente al Estado y a las demás instituciones y actores sociales. Los autores abordan el tema del capital social en la interpretación y análisis de la producción de hábitat popular y de la promoción del desarrollo urbano integral en la ciudad de Buenos Aires; desde esta perspectiva se desprende la hipótesis que la capacidad de movilización y disponibilidad del recurso vinculado al capital social implica necesariamente una apuesta de luchas en donde lo político juega un rol relevante. El “capital político" constituiría un elemento conceptual relevante a considerar tanto en el análisis como en los procesos de producción de hábitat popular. Este concepto englobaría un conjunto de recursos estratégicos que los movimientos sociales poseen para la generación de condiciones de eficacia en las intervenciones y acciones sociales "a escala" en los procesos de producción de hábitat popular y en la reducción de la pobreza y la desigualdad social. Las preguntas principales se refieren a lo que no se considera cuando se habla de Capital Social; qué es lo que si se considera cuando los movimientos sociales producen hábitat popular; y, qué está en juego en el Capital Político, ¿empowerment o valorización del Capital Social? El desarrollo y consolidación de un relevante capital político pone en valor, simbólicamente, el capital social mediante el desarrollo de su capacidad operativa, de la consecución de resultados concretos y de su sustentabilidad a largo plazo como actor urbano. Permite que las intervenciones de los movimientos sociales y las organizaciones de base en la producción de hábitat popular influyan en mayor escala y se constituyan en un agente de desarrollo urbano "desde abajo".

Ernesto Turner en su artículo "Desarrollo y Pobreza en México, Argentina, Brasil y Chile" señala que la pobreza en América Latina no es un hecho contemporáneo, sino que desde la colonia afectaba a gran parte de la población. Retomando algunos aspectos del caso mexicano, explica que son condiciones históricas las que dan pie a la brecha del crecimiento económico entre América Latina y Estados Unidos, así como la desigualdad de los ingresos. Esto lo ejemplifica con los casos de México, Argentina, Brasil y Chile, respecto de EUA, Japón y Alemania, presentando la evolución del PIB per cápita y la distribución del ingreso de 1980 a 2010. En seguida distingue la pobreza extrema y la moderada, donde en esta última el individuo no tiene acceso a los bienes comúnmente obtenidos por la mayoría de los individuos de su comunidad, pero sí puede satisfacer sus necesidades básicas. Como parte de la pobreza moderada desglosa la pobreza de capacidades y de patrimonio. Finalmente con base en dicho enfo- 
que de la pobreza, la analiza en México, Argentina, Brasil y Chile en las últimas décadas.

Finalmente en su trabajo "El misterio latinoamericano", Ricardo Yoselevsky y Óscar Cuéllar Saavedra recuperan a América Latina como una construcción intelectual que intenta dar cuenta de los procesos de desarrollo económico, entendiendo el corto alcance del "Consenso de Washington” como forma de integración al mundo capitalista, pero también como la ausencia de un programa de desarrollo nacional. Las distintas experiencias de izquierda que han sido el mayor esfuerzo de la región no constituyen una alternativa real -como sí lo fue Cuba en los años sesenta- ni desde el punto de vista de los programas ni desde el liderazgo, lo cual puede interpretarse como una derrota ideológica. Los modelos desarrollistas impulsados en las décadas de los cincuentas y sesentas por la CEPAL significaban una conciencia de la relevancia de un proyecto continental que merecía ser respaldado por la construcción de un objeto de conocimiento, al mismo tiempo que por un actor político colectivo, cuestión que hoy día parece ausente.

En la sección “Cartografías para el Futuro”, se han incluido los trabajos "Más allá de la Economía Ecológica, la construcción de nichos de sostenibilidad” de autoría de Pedro Medellín-Milán, José Antonio Avalos-Lozano y Luz María Nieto-Caraveo; y de María Eugenia Santana, el artículo titulado "Recrear el dinero en una economía solidaria”

La sección Proyectos y avances de investigación incluye los siguientes artículos: de Bernardo Castro Sáez “Aportes de Niklas Luhmann a la teoría de la complejidad"; de Eda Cleary "EI poder y los valores en Weber, Freud y Kelsen a la luz del "nuevo paradigma científico" y en relación con América Latina; de Claudio Gajardo Cornejo "Aproximación a la industria discográfica y su relación con la industria radial en Chile (1964-1967)”; de Luis García Fanlo “La argentinidad: un marco interpretativo”; de Clara Olmedo e Iñaki Ceberio “Antagonismo. Defensa del Medioambiente vs. Defensa de Fuentes de Trabajo”; de Salvador Peiró i Grègori y Gladys Merma Molina "Una mirada crítica a las repercusiones de la televisión en la educación”; y de Paulina Salinas y Jaime Barrientos "La discriminación de género y los modelos relacionales en la minería. Análisis de los discursos de las garzonas en las salas de cerveza en el norte de Chile”.

El número concluye con la sección Comentarios y reseñas de libros que presenta tres reseñas: la primera es de María Gabriela Córdova sobre el libro La Medición del Progreso y del Bienestar. Propuestas desde América Latina; Luis Eduardo Thayer escribe la reseña del libro Catástrofes medioambientales. La reacción social y la política de José Ignacio Cases (editor); y la última es realizada por Marcel Théza, quien comenta el libro La Naturaleza con derechos. De la filosofía a la política de Alberto Acosta y Esperanza Martínez (compiladores). 